\title{
Using Virtual Reality to Promote Physical Activity
}

\author{
Shuo Zhou1,2 (1) \\ ${ }^{1}$ Department of Community and Behavioral Health, Colorado School of Public Health, Aurora, CO, USA \\ ${ }^{2}$ Division of Cancer Prevention and Control, University of Colorado Cancer Center, Aurora, CO, USA \\ Email: shuo.zhou@cuanschutz.edu
}

How to cite this paper: Zhou, S. (2020) Using Virtual Reality to Promote Physical Activity. Journal of Software Engineering and Applications, 13, 312-326.

https://doi.org/10.4236/jsea.2020.1311021

Received: October 1, 2020

Accepted: November 27, 2020

Published: November 30, 2020

Copyright $\odot 2020$ by author(s) and Scientific Research Publishing Inc. This work is licensed under the Creative Commons Attribution International License (CC BY 4.0).

http://creativecommons.org/licenses/by/4.0/ (c) (i) Open Access

\begin{abstract}
The purpose of this research is to develop an immersive virtual fitness center as an intervention to promote physical activity and examine its impacts on users' exercise plans and exercise behaviors. The virtual reality (VR)-based intervention includes three main features: enabling the users to control their avatar, personalizing the avatar to look the same as the user, and visualizing the positive consequences of exercising. We conducted an experiment to randomly assign participants to two treatment groups: experiencing either the self or another person losing weight because of exercising in the virtual environment. The findings demonstrated that the self-avatar group exercised more in the voluntary section than the other-avatar group. However, participants in the self-avatar condition perceived a lower level of sense of presence compared to participants in the other-avatar condition. Compared to people in the control condition who watched and followed the exercise from a workout video, those who exercised in the virtual fitness center, regardless of whether the avatar was based on the self's image or another person's image, planned to spend more time on exercising in the following week. Theoretical and practical implications for using VR technology to promote health-related behavioral change, and why personalization decreases perceived sense of presence in the virtual environment are discussed.
\end{abstract}

\section{Keywords}

Virtual Reality, Personalization, Visualization, Presence, Physical Activity/Exercise

\section{Introduction}

The emerging technology of virtual reality (VR) provides us an opportunity to 
explore how to better engage people with health messages and apply this innovative method for promoting health behaviors and advancing new theories. Immersive virtual environment technology creates engrossing, believable, and highly controlled worlds that seem real to the users by substituting sensory information we receive in the real world with stereoscopic view of the virtual environment, sound feedback, and real-time synchronization of one's behaviors. As an advanced form of human-computer interface, VR enables users to have brand new physical, psychological, and social experiences by interacting with and immersing into the computer-generated virtual environments. Through VR, users can receive health treatments or education no longer as passive viewers, but as active participants in a high-fidelity, interactive, and personalized virtual world.

VR has been found to have several capabilities for health promotion. First, it empowers patients to successfully manage a problematic situation related to his/ her disturbance. For example, VR enables people to repeatedly expose to their feared stimulus in safe environments. This function has been used to treat posttraumatic stress disorder, anxiety disorders, and phobias through VR-based exposure therapy [1] [2] [3]. Second, it facilitates rehabilitation by motivating people to practice and perform certain movements through game-based design [4] [5]. Third, it distracts people's attention from the painful or depressed status by engaging patients into a novel fictional world [6] [7]. Last, it is also a useful research tool for performance assessment because it can monitor people's cognitive and motor responses in real time [8].

The current research focuses on one important application of VR-encouraging people to exercise and keep fit. Although an increasing number of VR interventions have been designed and applied in clinical settings to improve the amount of physical activity, balance, or muscle strength [9] [10] [11], investigations of specific features of the VR intervention and theoretical constructs that drive the intervention effectiveness are limited. Our goal is to 1) develop a theorydriven VR-based intervention, 2) examine its effects on motivating people to engage in physical activities, and 3) identify the underlying mechanisms of how the VR experience influences users' intention to exercise.

\section{Overcoming Barriers to Physical Activity}

Although there is mounting evidence for the health benefits of regular physical activity, about $40 \%$ of Americans self-reported that they are not active at recommended levels according to the Physical Activity Guidelines for Americans (PAGA). The proportion of US adults meeting the PAGA was even less $(<10 \%)$ measured by accelerometry [12]. Besides environmental barriers, lacking selfmotivation is the key reason for not engaging in physical activities. People tend to impulsively privilege immediate over delayed satisfaction of needs [13]. Since exercising and losing weight are long-term processes, people need to wait for several months or even longer to see the direct benefits of workouts. The change of body shape could not be shown instantly, making people not confident enough to 
achieve their weight-loss goals. The vague and uncertain consequences of workouts also discourage people from committing to a long-term exercise plan. Therefore, there is a need to present the positive consequences of exercising more directly and instantly to improve people's motivations to participate in physical activities.

\section{Using VR for Promoting Physical Activity}

Virtual reality technologies are useful tools to address barriers to exercising. VR provides user-centered experience that enables people to immerse into a virtual environment, for example, having a virtual tour of a fitness center through headmounted displays (HMD). Users can virtually explore the facilities in a gym and can choose which equipment they want to work on. The virtual world makes it possible to show the positive consequence of exercising vividly right in front of the user's eyes. The avatar or the main character of the VR experience can also be personalized to look similar to the user, which further enhances user engagement and establishes a close connection between the user and the virtual experience.

Social cognitive theory explains the mechanisms of how to motivate people for behavioral changes. It suggests that people do not need to personally try a new behavior, instead, we learn by observing others, with the environment, behavior, and personal cognition acting as primary factors influencing the learning process. When people observe a model performing a behavior, they remember the consequence of the events and use this information to guide their subsequent behaviors. This is also called the vicarious learning process, or vicarious reinforcement [14]. Observing positive consequences of a behavior will motivate people to model the behavior, whereas observing negative consequences of a behavior will discourage people from performing the behavior.

Vicarious learning has been widely applied to guide the design of health communication campaigns. In the specific context of promoting physical activity, for example, reading the testimony of a breast cancer survivor who had been physically active during and after treatment improved cancer patients' perceived selfefficacy and intentions to exercise compared to the informational and no message conditions [15]. It is also found that using gain frames by demonstrating the rewards of exercising is more effective in changing people's beliefs and motivating people to exercise compared to using loss frames [16].

To enhance the vicarious learning process, people should perceive themselves similar to the person being observed, or feel closely related to the observed person, which is termed identification. Identification with the model increases the viewers' attention to the health topic, self-referencing to the message, and personal involvement with the issue [17]. Perceived similarity between the self and the model also motivates observers to perform learned behaviors and increases their perceived self-efficacy in performing the observed behavior and receiving the same outcomes [14]. 
Similarity may be based on physical traits, personality, beliefs and attitudes, or pervious behaviors. Among them, physical features are the most immediate and direct determinants of perceived similarity. Abundant evidence shows physical similarity between audience members and the model influences the effectiveness of health communication messages. When viewers and the exemplar in a sun protection message are of the same gender, viewers rated the message more effective [18]. People preferred to choose avatars of the same ethnicity or look similar to them in an interactive computer-delivered video to prevent sexually transmitted infections [19], particularly among Hispanic and African American participants. African Americans also responded more favorably to Black (as opposed to White) spokespersons in a HIV public service announcement (PSA) and evaluated the PSA with Black spokespersons more positively [20]. However, except from Fox and Bailenson [21], few studies have tested the effects of highly personalized models tailored to each individual audience member. Virtual reality technology enables researchers to personalize avatars based on the appearance of each user, maximizing the level of identification with the person being observed.

Guided by this theoretical framework, we developed a VR-based intervention for promoting physical activity, which emphasizes on three important features of VR experience-1) visualizing the potential positive consequences of workout, 2) personalizing the avatar to look similar to the user, and 3) immersing the self into the virtual environment.

\subsection{Visualizing the Outcomes of Exercising}

We designed a VR program simulating the process of losing weight after working out. When users first enter the virtual fitness center, they are embodied in an obese avatar. After several sessions of exercising, the avatar loses weight with a fitter body shape, visualizing the positive outcomes of exercising.

According to social cognitive theory, observing the positive consequences of a behavior motivates people to model the same behavior whereas observing the negative consequences of a behavior discourages people to do the same thing. Vicariously experiencing the outcomes of a health behavior plays an important role in influencing people's subsequent behaviors. In the immersive virtual environment, such vicarious learning is enhanced by the realistic sensory feedback and high level of interactivity between the user and the virtual environment. If users could see how their physical bodies change after a certain period of training enabled by virtual reality technology, they should be more motivated to participate in physical activities. Therefore, we hypothesize that

$H 1$ : People experiencing weight loss in a virtual fitness center will engage in more physical activities than those without the virtual experience.

\subsection{Personalization}

The power of generating vivid, concrete, and personalized messages has been well documented in the field of communication and advertising [22] [23]. In our 
VR program, users can embody an avatar that is created based on their own image, which personalizes the virtual experience to the highest level. Customized avatar that looks the same as the user may enhance user's engagement into the virtual world, identification with the avatar, perceived self-relevance to the exercising activities, and perceived efficacy to achieve the intended outcomes demonstrated in the virtual world.

Users can see how the body of the "future self" would change and experience this change from the first-person view, making this VR experience more realistic and reducing people's psychological reactance. Therefore, we hypothesize that

H2: Participants embodied in a personalized avatar that looks the same as the participant will engage in more physical activities than those embodied in a stranger avatar that looks different from the participant.

\subsection{Immersive Virtual Experience}

Immersion is a description of a technology, capturing the extent to which computer displays can deliver an inclusive, extensive, surrounding, and vivid illusion of reality [24]. The more immersive people could get into the virtual world, the more impactful the virtual experience on user's perceptions, attitudes, and behaviors. Several constructs were conceptualized to capture people's immersive virtual experience, including egocentric projection, identification, presence, and perceived agency.

Egocentric projection is the extent to which a user imagines the self as the avatar in the virtual environment and projects his/her presumed thoughts or feelings in the virtual situation into the mind of the avatar. As explained in the earlier section about vicarious learning, identification is defined as an imaginative process through which users mentally simulate the avatar's activities in the virtual environment, imagine what the avatar is thinking and feeling, and adopt the avatar's goals, thoughts, and emotions [25]. Presence refers to the subjective experience of being in one place or environment, even when one is physically situated in another environment. And perceived agency is the perception about one's ability to control behaviors in the virtual environment.

The proposed VR program enhances users' immersive experience by enabling users to control their avatar using hand controllers, providing real-time synchronization of their behaviors in the virtual environment, and allowing them to try different exercise equipment in the virtual fitness center. This research tests whether immersion into the virtual world could explain how the overall VR intervention and the specific feature of avatar personalization affect people's motivation to exercise.

$H 3$ : a) Egocentric projection and b) identification will mediate the effect of virtual fitness center experience on motivating people to exercise.

H4: a) Egocentric projection, b) identification, c) presence, and d) perceived agency will mediate the effect of avatar personalization on motivating people to exercise. 


\section{Method}

The experiment utilized a between-subject design (see Table 1). Participants were randomly assigned to the two VR treatment conditions: the personalized condition in which the self was the avatar, or the non-personalized condition in which a stranger was the avatar in a virtual fitness center. We also included a no VR experience control condition in which participants watched and followed exercise from a workout video. Figure 1 demonstrates the scenes participants

Table 1. Experimental design.

\begin{tabular}{ccc}
\hline Condition $1(\mathrm{n}=13)$ & Condition 2 $(\mathrm{n}=14)$ & Condition 3 $(\mathrm{n}=15)$ \\
\hline VR Fitness Center Experience & VR Fitness Center Experience & 2D Workout Video \\
with Personalization & without Personalization & \\
\hline
\end{tabular}

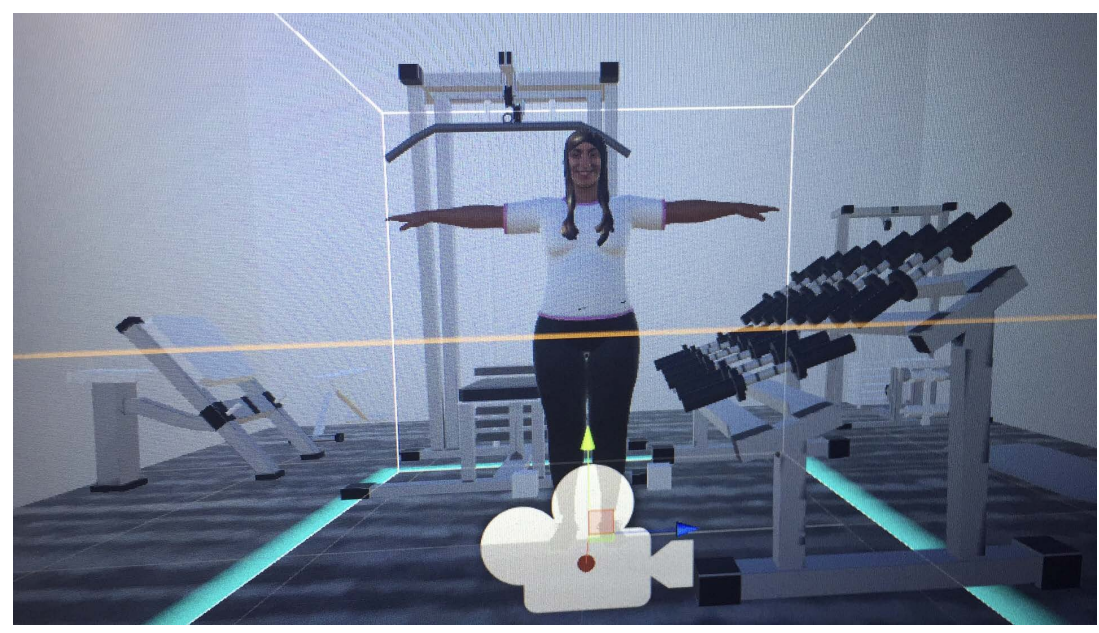

(a)
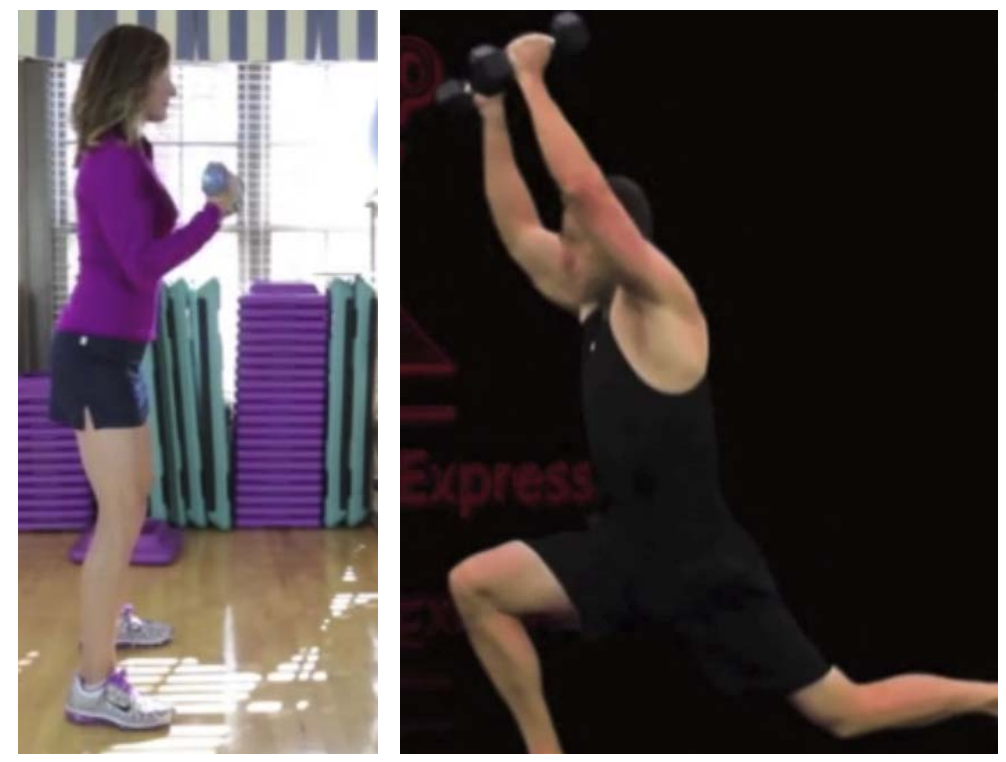

(b)

Figure 1. (a) Demonstration of the treatment condition: VR fitness center experience; (b) Demonstration of the control condition: workout video. 
saw in the VR treatment and control conditions.

\subsection{Participants}

Twenty-seven participants were recruited from a US university for the two VR treatment conditions. An additional 15 participants were recruited to complete an online survey through Qualtrics.com as the control condition. Each participant who completed the study was offered $\$ 10$ as the compensation for their participation. In total, the sample consisted of 42 participants. Among them, the majority were White Americans (64.3\%), followed by Asians (16.7\%), Latinos or Mexican Americans (9.5\%), and African Americans (7.1\%). There were 42.9\% male participants and $57.1 \%$ female participants. The average age was 33 ( $S D=$ 11.57).

\subsection{Manipulation \& Procedures}

Personalization of the avatar was manipulated by generating the avatar based on either the participant's own head photos (self-avatar) or a stranger's photos (other-avatar). The avatars in the personalized condition look the same as the participants themselves, whereas the avatars in the non-personalized condition look different from the participants. The avatars were created in Daz Studio with faces generated in FaceGen Artist and then rigged in Mixamo. In both treatment conditions, participants immersed into the virtual fitness center and can see how their avatar's body shape changes if the avatar keeps exercising.

When participants came to the lab, the researcher briefly described the procedure of the experiment, helped participants familiarize with the VR equipment, and taught them how to use the hand controllers. Participants were instructed to put on the headset and started their virtual experience in a fitness center. The virtual fitness center holds a variety of equipment, including weights, a back machine, a combination machine, an abdominal bench, a yoga mat, and etc. In the first scene, participants were embodied in the self-avatar or otheravatar with an obese figure. They were instructed to perform five sets of upper body exercises in front of a mirror in the virtual fitness center and repeat each exercise for six times, resulting 30 movements in total. In the second scene, participants entered the virtual fitness center again and were embodied in a fitter body after losing weight. Participants were instructed to freely choose the equipment, do the exercise they want, and feel free to leave the virtual fitness center at any time. The researcher counted the number of exercise repetition participants voluntarily performed in this scene. After the virtual experience, participants answered a questionnaire about their experience in the virtual fitness center, including egocentric projection, identification with the avatar, sense of presence, and perceived agency to control the movements. We also asked their exercise plan for the next week, specifically, how long they will spend on exercising and what types of activities they will perform. Demographic information, including age, ethnicity, height, and weights were asked at the end. 
In the control condition, participants were instructed to watch a 30-second workout video and follow the exercise from the video. The video shows a character keeps the weight-lifting exercise but did not show how the body changes as a result of exercising. After watching the video, participants answered the same set of questions as the participants in the VR treatment conditions. Questions about perceived agency and presence were excluded because they were not applicable to the scenarios in the control condition.

\subsection{Measures}

Egocentric projection. Seven close-ended questions captured the extent to which participants use their own mental states to interpret the avatar's mind. These items were generated based on Ames' egocentric projection scale [26]. Participants rated their agreement with each statement on a 7-point scale $(1=$ strongly disagree, 7 = strongly agree). Sample questions included, "The avatar has the same goals I would have in that situation"; "The avatar has the same skills I would have in that situation"; "My avatar is the same kind of person I would be in that situation"; "The avatar acted the same way I would act in that situation". The presentation order of the questions was randomized. The seven items were averaged into an egocentric projection scale (Cronbach's $\alpha=0.92, M$ $=4.25, S D=1.36$ ).

Identification. Identification was measured with a 10-item identification scale [25]. Items include statements about the relationship between the participant and the avatar (or the character in the control condition). For example, "I could really 'get inside' my avatar's head", "I think I understand my avatar well" and "I understood the events in the way my avatar understood them". Participants rated to what extent they agreed or disagreed with each statement on a 7-point scale (1 = strongly disagree, $7=$ strongly agree). The ten items were averaged into an identification scale (Cronbach's $\alpha=0.86, M=3.94, S D=0.98$ ).

Perceived Agency. Perceive agency was measured by four questions asking whether the participants believe they can control their actions in the virtual environment. The four items included "I felt I had control over my actions", "I did NOT have control over my actions" "I could fully control my actions", and "I could NOT control my actions very well". Participants rated to what extent they agreed or disagreed with each statement on a 7-point scale $(1=$ strongly disagree, 7 = strongly agree). Items two and four were reverse coded.

Reliability of the four items (Cronbach's $\alpha=0.63$ ) was lower than what is commonly recommended for a reliable scale $(>0.70)$. After removing the second item, the remaining three items formed a reliable scale. Thus, the three items were averaged into a perceived agency scale (Cronbach's $\alpha=0.71, M=5.30, S D$ $=1.04$ ).

Sense of Presence. Sense of presence includes three dimensions: spatial sense of presence, self sense of presence, and social sense of presence [27]. The social dimension is not applicable to this study because there was only one avatar in 
the virtual environment and no social interactions were involved in any scenarios. Example items were "The avatar's body was my own body", "I was in the avatar's body", "The avatar was an extension of me", "I felt surrounded by the virtual room", and "I really visited the virtual room". Participants rated to what extent they agreed or disagreed with each statement on a 7-point scale $(1=$ strongly disagree, $7=$ strongly agree). The ten items were averaged into a presence scale (Cronbach's $\alpha=0.85, M=4.08, S D=0.86$ ).

Intention to exercise. Participants' intention to exercise was measured using a single question asking how many hours and minutes they plan to spend next week to participate in sports and other vigorous physical activities, excluding walking and time spent in required school physical education classes. Participants input the exact numbers of hours and minutes of their exercise plan and the total time was transformed into hours $(M=3.45, S D=2.34)$.

Types of physical activity. The number of different types of physical activity was measured by asking participants to check all the activities they plan to do in the next week, including running, hiking, bicycling, swimming etc. They can choose multiple items $(M=3.12, S D=1.88)$.

Exercise repetitions. The researcher observed and counted the number of exercise repetition participants voluntarily performed in the second scene in the two VR treatment conditions $(M=23.44, S D=12.86)$.

\section{Results}

\subsection{Effects of VR Experience on Motivation to Exercise}

Hypothesis 1 predicts that virtually experience losing weight in a fitness center will motivate people to engage in more exercises. We compared the means of hours participants intend to do physical activities in the next week among those in the two VR treatment conditions to those in the control condition who watched a workout video. All the analyses were conducted using the SPSS Statistics 24 . Result of an independent samples t-test showed the main effect of the virtual experience on exercise intention was significant, $t(40)=3.16, p=0.003$. People who received the VR-based intervention were more motivated to engage in physical activities in the following week $(M=4.22, S E=0.45)$ than people who only watched the video $(M=2.07, S E=0.43)$. This effect was mainly driven by the difference between the self-avatar condition and the control condition (Mdifference $=2.66, S E=0.80, p=0.007)$. People who saw their own avatars getting fitter in the virtual fitness center planned to allocate the longest time to exercise ( $M$ $=4.73, S D=2.69)$, followed by the other-avatar VR condition $(M=3.75, S D=$ 1.92). People who simply watched the workout video were least motivated to exercise in the next week $(M=2.07, S D=1.66)$ (see Figure 2). Thus hypothesis 1 is supported.

In addition, participants who had the VR fitness center experience were likely to participate in more diverse types of physical activities $(M=3.48, S E=0.39)$, including swimming, basketball, walking, running, tennis, etc., than those who 
did not see the positive consequences of exercising in workout videos $(M=2.47$, $S E=0.36)$ at a marginally significant level, $t(40)=1.72, p=0.09$.

\subsection{Effects of Personalization on Actual Exercising Behavior}

Hypothesis 2 predicts that personalizing the avatar to be similar to the participant will increase participants' motivation to exercise and make people more likely to engage in physical activities. Result of a t-test showed a significant main effect of personalization on exercise repetition in the voluntary exercise session, $t(25)$ $=4.64, p<0.001$. Participants in the self-avatar condition performed more sets of exercises $(M=32.23, S E=3.54)$ than participants in the other-avatar condition $(M=15.29, S E=1.28)$ (see Figure 3). Therefore, hypothesis 2 is supported.

\subsection{Testing the Role of Immersion}

Hypotheses 3 and 4 predict that constructs measuring people's immersive virtual experience including egocentric projection, identification, presence, and perceived agency can explain how the VR experience and personalization affect people's motivation to exercise. A set of ANOVAs were performed to test $\mathrm{H} 3$ and H4. The main effects of conditions on egocentric projection, identification, and perceived agency were not significant. Thus hypotheses 3 and 4 were rejected. However, we found a significant effect of personalization on presence, $F$ $(1,25)=5.75, p=0.024, \eta_{p}^{2}=0.19$. Contradictory to our prediction, participants in the self-avatar condition reported lower level of presence $(M=3.98, S E$ $=0.27)$ than participants in the other-avatar condition $(M=4.84, S E=0.24)$.

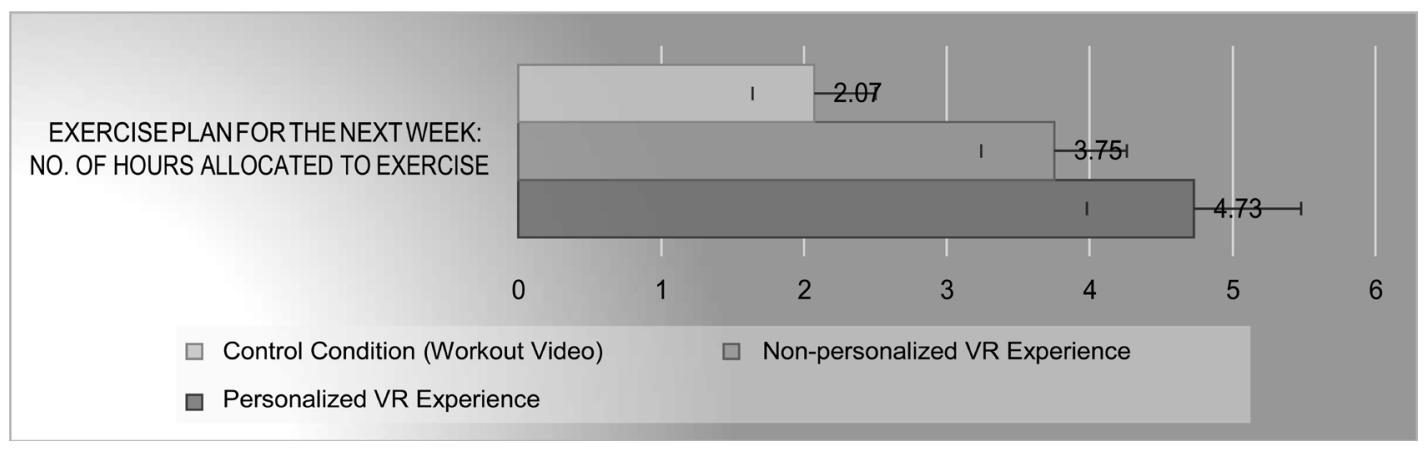

Figure 2. Conditions on the number of hours planned for exercising.

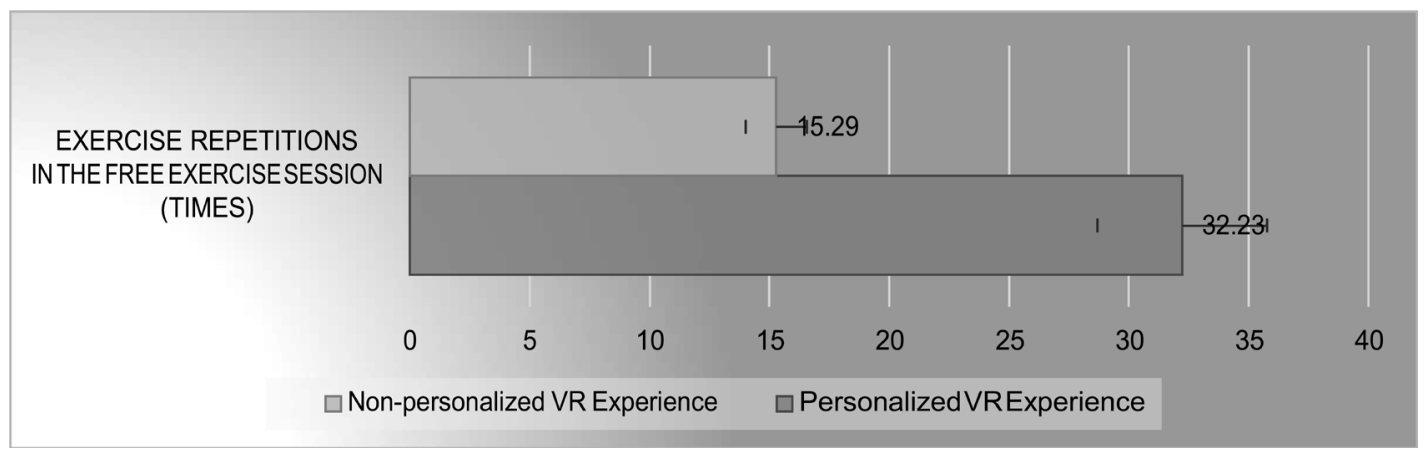

Figure 3. Effect of personalization on exercise repetitions in the free exercise session. 


\section{Discussion}

This study developed an immersive virtual fitness center and examined its impacts on motivating people's exercising behaviors and plans. It shows that seeing an avatar losing weight in a virtual fitness center, regardless of whether the avatar is based on the self or the other person's image, increased people's intention to spend longer time on exercising compared to simply watching and following a workout video. The experiment also suggests that personalizing the avatar to look similar to the user encouraged the user to do more exercise in the voluntary exercise session compared to not personalizing the avatar.

\subsection{Practical Implications}

Through virtual reality technology, we can generate self-like avatars to demonstrate the potential consequences of health behaviors vividly and instantly, maximizing the effects of vicarious reinforcement and identification on behavioral change. Vicarious reinforcement and identification are two critical components of the social cognitive theory. Traditional ways of manipulating vicarious reinforcement include using gain or loss frame, explicitly describing the positive or negative consequences of a behavior, or embedding the rewards or punishments in a story. Instead of witnessing what happened to another person from an observer's perspective, virtual reality enables the user to virtually experience the outcomes of a health behavior from the actor's perspective-actively participated in the behavior change process. Although we did not find significant differences in participants' levels of egocentric projection or identification with the avatar or character between virtual reality experience and video watching, immersion into the virtual fitness center was more effective in motivating people to exercise. Studies may further explore the mechanisms of how virtual reality experience influences people's health beliefs and subsequent behaviors, for example, whether the virtual content is more powerful than traditional methods in enhancing people's perceived realism, self-relevance, and transportation into the behavioral event.

Virtual reality technology also maximizes the similarity between the user and the avatar by generating an avatar based on each individual's image. Personalization at the individual level is stronger than simply matching the sex, ethnicity, or personality between the user and the observed model. Seeing the benefits of self-avatars doing physical activity makes people voluntarily exercise more than seeing the benefits of other-avatars. Surprisingly, the personalization manipulation did not influence people's identification with the avatar, indicating the effect of personalizing the avatar on behavioral change was not through increased perceived similarity, or self-reported identification as suggested in the social cognitive theory. One possible explanation is that exposure to self-avatar's movements makes people more likely to unconsciously mimic the same behavior instead of consciously affecting people's engagement with the avatar or motivation to exercise. This is consistent with our finding that personalization only in- 
fluenced people's actual behavior in the free-exercise session but not people's intention to exercise in the week following the experiment. According to the vicarious goal contagion theory, people would unconsciously adopt the goal of another person when observing the person performing a behavior, especially when they are similar to the person [28]. Similarity here refers to the manipulated similarity rather than the self-report measure of perceived similarity. It is worth further examining whether the personalization effect on health-related behavioral change is driven by unconscious processes and how long the effect will last.

A surprising but interesting finding is that although personalizing the avatar increased behavioral outcomes, it tends to decrease people's sense of presence in the virtual environment. It may be because people pay closer attention to the self-avatar's behaviors than other-avatar's behaviors since they are more self-relevant. When exposed to an avatar based on their own image, people may set higher standards for the presentation of the avatar's appearance, require greater precision in the synchronization between their real and virtual movements, and easily identify the differences between the self-based avatar and the actual self. Minor deviations from their real images or movements may induce strong feelings of discomfort, disliking, or resistance. One extension of the current study could be investigating people's attitudes toward and evaluations of the personalized avatar and identifying the key components of personalization to maximize its effects on behavioral change in the health contexts.

\subsection{Limitations}

The current study has some limitations. First, the experiment was not a fully randomized design. Although we randomly assigned participants to the two VR treatment conditions, participants in the control condition were recruited at a later stage. Therefore, cautions should be taken when comparing between the treatment and control conditions. The effects of the VR intervention may be confounded by factors related to characters of the sample, self-selection bias, participants' past experience of exercising, or the time of recruitment.

Second, we only explored the overall effect of the VR experience and the specific impact of avatar personalization. Other unique features of the immersive VR experience may also drive the effect of the VR-based intervention for physical activity promotion, for example, feeling of control over the avatar's behavior, visualizing the consequences, having freedom to choose equipment, being able to navigate the virtual environment. Future studies are encouraged to explore the underlying mechanisms beyond avatar personalization using a fully controlled factorial design.

Last, the current study only provided visual and audio feedback to the participants. People cannot feel the weight of the dumbbells. Participants picked up the weights by pressing the trigger on the left- and right-hand controllers. Although participants had the option to choose weights ranging from $5 \mathrm{lbs}$ to 30 
lbs, the dumbbells did not reflect their actual weights. Future studies may attach sensors to real dumbbells to enhance the realism of the virtual experience and maximize its effects on changing health-related behaviors.

\section{Conclusion}

Virtually experiencing the self losing weight after exercising is an effective strategy to motivate people to engage in physical activities. Through an experiment, we found that people seeing the self-avatar losing weight in a virtual fitness center exercised more in the voluntary session than people seeing the other-avatar losing weight. In addition, compared to people watching and following an exercising video, those who virtually experienced weight-loss after exercising, regardless of whether the avatar was based on the self's or another person's image, planned to spend more time on exercising in the following week. The immersive virtual environment technology can be an innovative and useful tool for motivating health behaviors and promoting public health.

\section{Fund}

This research was partially funded by the HCT Vive Research Fund.

\section{Conflicts of Interest}

The author declares no conflicts of interest regarding the publication of this paper.

\section{References}

[1] Parsons, T.D. and Rizzo, A.A. (2008) Affective Outcomes of Virtual Reality Exposure Therapy for Anxiety and Specific Phobias: A Meta-Analysis. Journal of Behavior Therapy and Experimental Psychiatry, 39, 250-261. https://doi.org/10.1016/j.jbtep.2007.07.007

[2] Powers, M.B. and Emmelkamp, P.M. (2008) Virtual Reality Exposure Therapy for Anxiety Disorders: A Meta-Analysis. Journal of Anxiety Disorders, 22, 561-569. https://doi.org/10.1016/j.janxdis.2007.04.006

[3] Wiederhold, B.K., Jang, D.P., Gevirtz, R.G., et al. (2002) The Treatment of Fear of Flying: A Controlled Study of Imaginal and Virtual Reality Graded Exposure Therapy. IEEE Transactions on Information Technology in Biomedicine, 6, 218-223. https://doi.org/10.1109/TITB.2002.802378

[4] Jack, D., Boian, R., Merians, A.S., et al. (2001) Virtual Reality-Enhanced Stroke Rehabilitation. IEEE Transactions on Neural Systems and Rehabilitation Engineering, 9, 308-318. https://doi.org/10.1109/7333.948460

[5] Rizzo, A.S. and Kim, G.J. (2005) A SWOT Analysis of the Field of Virtual Reality Rehabilitation and Therapy. Presence: Teleoperators \& Virtual Environments, 14, 119-146. https://doi.org/10.1162/1054746053967094

[6] Hoffman, H.G., Patterson, D.R. and Carrougher, G.J. (2000) Use of Virtual Reality for Adjunctive Treatment of Adult Burn Pain during Physical Therapy: A Controlled Study. The Clinical Journal of Pain, 16, 244-250. https://doi.org/10.1097/00002508-200009000-00010

[7] Malloy, K.M. and Milling, L.S. (2010) The Effectiveness of Virtual Reality Distrac- 
tion for Pain Reduction: A Systematic Review. Clinical Psychology Review, 30, 10111018. https://doi.org/10.1016/j.cpr.2010.07.001

[8] Parsons, T.D. and Rizzo, A.A. (2008) Initial Validation of a Virtual Environment for Assessment of Memory Functioning: Virtual Reality Cognitive Performance Assessment Test. CyberPsychology \& Behavior, 11, 17-25.

https://doi.org/10.1089/cpb.2007.9934

[9] Cho, H. and Sohng, K.-Y. (2014) The Effect of a Virtual Reality Exercise Program on Physical Fitness, Body Composition, and Fatigue in Hemodialysis Patients. Journal of Physical Therapy Science, 26, 1661-1665. https://doi.org/10.1589/jpts.26.1661

[10] Park, J., Lee, D. and Lee, S. (2014) Effect of Virtual Reality Exercise Using the Nintendo WII Fit on Muscle Activities of the Trunk and Lower Extremities of Normal Adults. Journal of Physical Therapy Science, 26, 271-273.

https://doi.org/10.1589/jpts.26.271

[11] Park, E.-C., Kim, S.-G. and Lee, C.-W. (2015) The Effects of Virtual Reality Game Exercise on Balance and Gait of the Elderly. Journal of Physical Therapy Science, 27, 1157-1159. https://doi.org/10.1589/jpts.27.1157

[12] Tucker, J.M., Welk, G.J. and Beyler, N.K. (2011) Physical Activity in US Adults: Compliance with the Physical Activity Guidelines for Americans. American Journal of Preventive Medicine, 40, 454-461. https://doi.org/10.1016/j.amepre.2010.12.016

[13] Zhang, B., Wu, M., Kang, H., et al. (2014) Effects of Security Warnings and Instant Gratification Cues on Attitudes toward Mobile Websites. Proceedings of the SIGCHI Conference on Human Factors in Computing Systems, 111-114. https://doi.org/10.1145/2556288.2557347

[14] Bandura, A. (1998) Health Promotion from the Perspective of Social Cognitive Theory. Psychology and Health, 13, 623-649. https://doi.org/10.1080/08870449808407422

[15] Falzon, C., Radel, R., Cantor, A., et al. (2015) Understanding Narrative Effects in Physical Activity Promotion: The Influence of Breast Cancer Survivor Testimony on Exercise Beliefs, Self-Efficacy, and Intention in Breast Cancer Patients. Supportive Care in Cancer, 23, 761-768. https://doi.org/10.1007/s00520-014-2422-x

[16] Gray, J.B. and Harrington, N.G. (2011) Narrative and Framing: A Test of an Integrated Message Strategy in the Exercise Context. Journal of Health Communication, 16, 264-281. https://doi.org/10.1080/10810730.2010.529490

[17] Kruglanski, A.W., Chen, X., Pierro, A., et al. (2006) Persuasion According to the Unimodel: Implications for Cancer Communication. Journal of Communication, 56, S105-S122. https://doi.org/10.1111/j.1460-2466.2006.00285.x

[18] Andsager, J.L., Bemker, V., Choi, H.-L., et al. (2006) Perceived Similarity of Exemplar Traits and Behavior: Effects on Message Evaluation. Communication Research, 33, 3-18. https://doi.org/10.1177/0093650205283099

[19] Ito, K.E., Kalyanaraman, S., Ford, C.A., et al. (2008) “Let's Talk about Sex": Pilot Study of an Interactive CD-ROM to Prevent HIV/STIS in Female Adolescents. AIDS Education and Prevention, 20, 78-89. https://doi.org/10.1521/aeap.2008.20.1.78

[20] Wang, X. and Arpan, L.M. (2008) Effects of Race and Ethnic Identity on Audience Evaluation of HIV Public Service Announcements. The Howard Journal of Communications, 19, 44-63. https://doi.org/10.1080/10646170701802019

[21] Fox, J. and Bailenson, J.N. (2009) Virtual Self-Modeling: The Effects of Vicarious Reinforcement and Identification on Exercise Behaviors. Media Psychology, 12, 1-25. https://doi.org/10.1080/15213260802669474

[22] Riddle, K. (2013) Transportation into Vivid Media Violence: A Focus on Attention, 
Emotions, and Mental Rumination. Communication Quarterly, 61, 446-462. https://doi.org/10.1080/01463373.2013.799512

[23] Zhou, S. and Niederdeppe, J. (2017). The Promises and Pitfalls of Personalization in Narratives to Promote Social Change. Communication Monographs, 84, 319-342. https://doi.org/10.1080/03637751.2016.1246348

[24] Slater, M. and Wilbur, S. (1997) A Framework for Immersive Virtual Environments (FIVE): Speculations on the Role of Presence in Virtual Environments. Presence: Teleoperators \& Virtual Environments, 6, 603-616. https://doi.org/10.1162/pres.1997.6.6.603

[25] Cohen, J. (2001) Defining Identification: A Theoretical Look at the Identification of Audiences with Media Characters. Mass Communication \& Society, 4, 245-264. https://doi.org/10.1207/S15327825MCS0403 01

[26] Ames, D.R. (2004) Inside the Mind Reader's Tool Kit: Projection and Stereotyping in Mental State Inference. Journal of Personality and Social Psychology, 87, 340353. https://doi.org/10.1037/0022-3514.87.3.340

[27] Lee, K.M. (2004) Presence, Explicated. Communication Theory, 14, 27-50. https://doi.org/10.1111/j.1468-2885.2004.tb00302.x

[28] Zhou, S., Shapiro, M.A. and Wansink, B. (2017) The Audience Eats More If a Movie Character Keeps Eating: An Unconscious Mechanism for Media Influence on Eating Behaviors. Appetite, 108, 407-415. https://doi.org/10.1016/j.appet.2016.10.028 